\title{
SUBSPACES OF BASICALLY DISCONNECTED SPACES OR QUOTIENTS OF COUNTABLY COMPLETE BOOLEAN ALGEBRAS
} BY

\author{
ERIC K. VAN DOUWEN ${ }^{1}$ AND JAN VAN MILL ${ }^{2}$
}

\begin{abstract}
Under MA $+2^{\omega}=\omega_{2}$ there is a (compact) strongly zero-dimensional $F$-space of weight $2^{\omega}$ which cannot be embedded in any basically disconnected space.

Dually, under MA $+2^{\omega}=\omega_{2}$ there is a weakly countably complete (or almost $\sigma$-complete, or countable separation property) Boolean algebra of cardinality $2^{\omega}$ which is not a homomorphic image of any countably complete Boolean algebra.

The key to our construction is the observation that if $X$ is a subspace of a basically disconnected space and $\beta \omega \subseteq X$ then $\beta \omega$ is a retract of $X$.

Dually, if $B$ is a homomorphic image of a countably complete Boolean algebra, and if $h$ is a homomorphism from $B$ onto $\mathscr{P}(\omega)$, the field of subsets of $\omega$, then there is an embedding $e: \mathscr{P}(\omega) \rightarrow B$ such that $h \circ e=\mathrm{id}_{\mathscr{P}(\omega)}$.
\end{abstract}

1. Introduction. In order not to double the length of this paper we will not dualize each statement; the basic translations can be found in the abstract and in $\$ 2$. The Boolean algebraic reader may assume that all spaces mentioned in this introduction are compact (see \$2) and read "zero-dimensional" for "strongly zero-dimensional".

It is well known that $\beta \omega$ is extremally disconnected, but that its closed subspace $\beta \omega-\omega$ is not even basically disconnected [GJ, 6W.3]. However, $\beta \omega-\omega$ is a zero-dimensional $F$-space. Since, more generally, the property of being a normal $F$-space is closed hereditary, this suggests consideration of the following statement.

$F E$ : Every strongly zero-dimensional $F$-space can be embedded in an Extremally disconnected space.

It is convenient to factor $F E$ as $F B+B E$, where

$F B$ : Every strongly zero-dimensional $F$-space can be embedded in a Basically disconnected space.

$B E$ : Every Basically disconnected space can be embedded in an Extremally disconnected space.

The earliest reference we are aware of where one of these statements is considered is [L]. Here Louveau attributes the question (or conjecture, Louveau is not clear) of whether $F E$ holds to Choquet, and proves that under $\mathrm{CH}$ the restriction of

Received by the editors December 20, 1978.

AMS (MOS) subject classifications (1970). Primary 06A40, 54C15, 54C25, 54G05; Secondary 02 K05. Key words and phrases. Strongly zero-dimensional, Boolean algebra, basically disconnected, countably complete, $F$-space, weakly countably complete, subspace, quotient, retraction.

${ }^{1}$ Partially supported by NSF Grant MCS78-09484.

'Supported by the Netherlands Organization for the Advancement of Pure Research (Z.W.O.), Juliana van Stolberglaan 148, 's-Gravenhage, The Netherlands. 
$F E$ to compact spaces of weight $\leqslant \mathrm{c}$ holds, i.e. $\mathrm{CH}$ implies

$\left.{ }^{*}\right)$ : every compact zero-dimensional $F$-space of weight $<c$ can be embedded in an extremally disconnected space.

The question of whether $B E$ holds was raised by Koppelberg, [K] (in Boolean algebraic language), who was apparently unaware of Louveau's paper. She proved that the restriction of $B E$ to compact spaces of weight $<\mathrm{c}$ holds under $\mathrm{CH}$.

The question of whether $F B$ holds was raised by van Douwen, Monk and Rubin, [vDMR], who also repeated the question of whether $B E$ holds.

In this paper we prove that $F B$ is not a theorem of $\mathrm{ZFC}$, hence neither is $F E$. We have no information about $B E$.

EXAMPLE (MA $\left.+c=\omega_{2}\right)$. There is a strongly zero-dimensional $F$-space $V$ which cannot be embedded in a basically disconnected space, such that $\beta V$ has weight $c$.

This example also shows that $\left(^{*}\right)$ is not a theorem of ZFC. In view of the fact that several results about $F$-spaces which had been proved under $\mathrm{CH}$ actually are equivalent to $\mathrm{CH}$, cf. [ $\mathrm{vD}_{1}$ ], [vDvM], we conjecture that $\left(^{*}\right)$ is equivalent to $\mathrm{CH}$. However, we need more than $\neg \mathrm{CH}$ for our construction since $\beta V$ "really" has weight $2^{\omega_{1}}$.

Our example is based on the following.

LEMMA. If $X$ can be embedded in a basically disconnected space, then $X$ has the property that every subspace of $X$ which is homeomorphic to $\beta \omega$ is a retract of $X$.

We build $V$ with $\beta \omega \subseteq V$. In order to ensure that $\beta \omega$ is not a retract of $V$ we badly need $\neg \mathrm{CH}$, and we do not know how to avoid it. We use MA $+c=\omega_{2}$ to make $V$ a strongly zero-dimensional $F$-space. We could have used less, but we do not bother because it does not help to get a real example, and one needs more than $\neg \mathrm{CH}$ to make $\beta V$ a counterexample to $\left({ }^{*}\right)$.

REMARK (ADDED IN PROOF). Because there seems to be a misunderstanding about the role of the hypothesis $c=\omega_{2}$ we mention the $V$ can be constructed under $\mathrm{MA}+\mathrm{c}=\kappa^{+}$with $\kappa$ any regular uncountable cardinal.

2. Preliminaries. As usual, an ordinal is the set of smaller ordinals and a cardinal is an initial ordinal.

Some of the definitions below are equivalent to the original definitions only in case of normal spaces but we will not bother. (This explains why in certain statements we have to hypothesize normality.) All our spaces are $T_{1}$. Clopen means closed and open. A space is called zero-dimensional if its clopen sets are a base, strongly zero-dimensional if any two disjoint closed sets are included in disjoint clopen sets. Recall that if $X$ is normal then $X$ is strongly zero-dimensional iff $\beta X$ is (strongly) zero-dimensional.

A space is called

extremally disconnected / basically disconnected/an F-space

if any two disjoint open sets

without further condition/one of which is an $F_{\sigma} /$ both of which are $F_{\sigma}$ 
have disjoint closures. Note that a normal space $X$ has one of these three properties iff $\beta X$ has it. (Normality is not needed for extremal disconnectedness.) Also note that the method of proof that a Lindelöf space is normal can be used to show that a normal space is an $F$-space (if and) only if any two $F_{\sigma}$-subsets $F$ and $G$ with $\bar{F} \cap G=F \cap \bar{G}=\varnothing$ have disjoint closures, (see e.g. [ $\left[\mathbf{v D}_{2}\right]$ for the easy proof). This implies that the property of being a normal $F$-space is closed hereditary. We also point out to the Boolean algebraic reader that although a normal basically disconnected space is strongly zero-dimensional, the analogous statement for $F$-spaces is false: Gillman and Henriksen give an example of a compact connected $F$-space in [GH, 2.8].

A BA (= Boolean algebra) will be identified with its universe. A BA B is called complete / countably complete / weakly countably complete

if for any two subsets $P$ and $Q$ such that $p \wedge q=0$ for $p \in P, q \in Q$ without further condition/with $|P|=\omega$ or $|Q|=\omega /$ with $|P|=|Q|=\omega$ there is an $s \in B$ which separates $P$ and $Q$, i.e. $p<s$ for $p \in P$ and $q<s^{\prime}$ for $q \in Q$. (Obviously this is equivalent to the usual definition of complete and countably complete.) It is well known and easy to prove that if $X$ is a zero-dimensional compact space, then $X$ is

extremally disconnected/basically disconnected/an $F$-space

if and only if the clopen algebra of $X$ is

complete/countably complete/weakly countably complete.

It is important to realize that in fact this is true if $X$ is strongly zero-dimensional, whether compact or not. This explains why we do not have to make our example $V$ compact, once we have $V$ we also have a compact example: just replace $V$ by $\beta V$.

\section{Proof of the Lemma.}

Topological PROOF. We may assume $X$ itself is basically disconnected, hence that $X$ is compact (for $\beta X$ is basically disconnected since $X$ is). Choose pairwise disjoint clopen $B_{n}$ 's with $n \in B_{n}$ for $n \in \omega$. Then $r=\cup_{n} B_{n} \times\{n\}$ is a retraction $\cup_{n} B_{n} \rightarrow \omega$. Let $A=\left(\cup_{n} B_{n}\right)^{-}$. Then $A$ is open and $A=\beta\left(\cup_{n} B_{n}\right)$ since $\cup_{n} B_{n}$ is an open $F_{\sigma}$. Hence $\beta r \cup(X-A) \times\{0\}$ is a retraction $X \rightarrow \beta \omega$.

Boolean AlgEBraic PROOF. Let $B$ be a countably complete BA, let $C$ be a BA, let $\mathscr{P}(\omega)$ be the field of subsets of $\omega$, and let

$$
g: B \rightarrow C \text { and } f: C \rightarrow \mathscr{P}(\omega)
$$

be homomorphisms onto. Choose disjoint $b_{n}$ 's in $B$ with $f(g(n))=n$ for $n \in \omega$, and let $a=\bigvee_{n} b_{n}$. Then we can define an embedding $e: \mathcal{P}(\omega) \rightarrow C$ such that $f \circ e=$ $\operatorname{id}_{\mathscr{P}(\omega)}$ by

$$
e(Y)= \begin{cases}g\left(\bigvee_{n \in Y} b_{n}\right) & \text { if } 0 \notin Y, \\ g\left(\left(\bigvee_{n \in Y} b_{n}\right) \vee a^{\prime}\right) & \text { if } 0 \in Y .\end{cases}
$$

4. The example. We want to use the Lemma to guarantee that our example cannot be embedded into a basically disconnected space. So we aim at adding a set $I$ to $\beta \omega$ and at topologizing $V=\beta \omega \cup I$ (with $I \cap \beta \omega=\varnothing$ ) in such a way that 
(1) $\beta \omega$ is not a retract of $V$.

We achieve (1) by a brute force application of $\neg \mathrm{CH}$, as the next fact makes clear. (Note that (2), (3) and (4) imply $\neg \mathrm{CH}$ since (4) forces $|I|>\omega_{1}$.)

FACT 1. Assume $\beta \omega-\omega$ has a subset $C$ such that

(2) there is an open family $\mathcal{Q}=\left\{U_{x}: x \in C\right\}$ in $\beta \omega-\omega$ such that $x \in U_{x}$ for $x \in C$ and $U_{x} \cap U_{y}=\varnothing$ for distinct $x, y \in C$,

(3) $|I<| C \mid$ and

(4) if $G$ is a $G_{\delta}$-set in $V$ with $C \subseteq G$, then $C \subseteq \overline{G \cap I}$.

Then $\beta \omega$ is not a retract of $V$.

Suppose there is a retraction $r: V \rightarrow \beta \omega$. Then $G=r^{\leftarrow}(\beta \omega-\omega)$ is a $G_{\delta}$-set in $V$ with $C \subseteq G$. So if $J=G \cap I$ then $C \subseteq \bar{J}$, therefore $C(=r \rightarrow C) \subseteq(r \rightarrow J)^{-}$. But $r \rightarrow J \subseteq r \rightarrow G=\beta \omega-\omega$, hence $(r \rightarrow J) \cap U_{x} \neq \varnothing$ for $x \in C$. This is impossible since $U_{x} \cap U_{y}=\varnothing$ for distinct $x, y \in C$ and $|r \rightarrow J|<|I|<|C|$.

Let $I$ be a set of cardinality $\omega_{1}$, and let $C$ be a subset of $\beta \omega-\omega$ of cardinality $\omega_{2}$, to be specified later. We plan to choose, for each $x \in C$, an uncountable subset $F_{x}$ of $I$ such that

(5) $\left|F_{x} \cap F_{y}\right| \leqslant \omega$ for distinct $x, y \in C$.

Again we specify our choice later. Let $T=I \cup C$ and topologize $T$ as follows: points of $I$ are isolated, a basic neighborhood of $x \in C$ has the form

$$
\{x\} \cup\left(F_{x}-K\right) \text { with }|K|<\omega .
$$

This is a valid neighborhood assignment because of (5). Evidently $T$ is a (regular) $P$-space. Topologize $V$ by pasting $T$ and $\beta \omega$ together, or, formally, $U \subseteq V$ is open in $V$ if $U \cap \beta \omega$ is open in $\beta \omega$ and $U \cap T$ is open in $T$. It is clear that (3) and (4) hold. The natural way to make (2) hold is to start with a disjoint open family $\mathscr{U}$ in $\beta \omega-\omega$ with $|\mathcal{Q}|=\omega_{2}$, choose $c(U) \in U$ for $U \in \mathcal{U}$ and let $C=\{c(U): U \in$ $\mathscr{U}$ \}. Unfortunately it is not clear that $V$ is going to be normal, or even Hausdorff, unless we could assert that $T$ is normal. However, we have the following fact.

FACT 2. $[\neg \mathrm{CH}]$ : No P-space which has a dense subset of cardinality $\omega_{1}$ and a closed discrete subset of cardinality $\omega_{2}$ is normal.

We prove this fact, which we obviously do not need for the construction of $V$, in the Appendix.

Note that both Facts 1 and 2 depend on the way we added $I$ to $\beta \omega$, and that apparently one cannot get Fact 1 without also getting Fact 2 . We overcome this difficulty by assuming MA.

Under MA $+c=\omega_{2}$ there is a point $p \in \beta \omega-\omega$ which has a clopen neighborhood base $\left\langle P_{\xi}: \xi<\omega_{2}\right\rangle$ in $\beta \omega$ such that

(6) if $\xi<\eta<\omega_{2}$ then $P_{\eta}-\omega \subset P_{\xi}-\omega$.

( $\subset$ denotes proper inclusion.) Choose $c_{\xi} \in\left(P_{\xi}-P_{\xi+1}\right)-\omega$ for $\xi<\omega_{2}$ and let $C=\left\{c_{\xi}: \xi<\omega_{2}\right\}$. Then clearly (2) holds. Also

(7) if $U$ is a neighborhood of $p$ then $|C-U|<\omega_{1}$.

It is precisely this fact which makes our construction work. For even though $T$ is not normal we do have

FACT 3. We can construct $T$ in such a way that for each $A \subseteq C$ with $|A|<\omega_{1}$ there is a clopen $S \subseteq T$ with $S \cap C=A$. 
We modify a construction of $\left[\mathbf{v D}_{3}\right]$. Let

$$
I=\omega_{1} \times \omega_{1}, \quad \Phi=\left\{f \subseteq \omega_{1} \times \omega_{1}: f \text { is a function from } \omega_{1} \text { to } \omega_{1}\right\} .
$$

Define a partial order $<^{*}$ on $\Phi$ by

$$
f<{ }^{*} g \text { if }\left|\left\{\xi<\omega_{1}: f(\xi)>g(\xi)\right\}\right|<\omega .
$$

With an easy induction, pick $f_{\xi} \in \Phi$ for $\xi<\omega_{2}$ such that

(8) if $\xi<\eta$ then $f_{\xi}<{ }^{*} f_{\eta}$.

For $x=c_{\xi} \in C$ we let $F_{x}=f_{\xi}$. Clearly (5) holds. To see that Fact 3 holds, let $A \subseteq \omega_{2}$ have cardinality $<\omega_{1}$. Let $\alpha=\sup (A)$. Then $\alpha<\omega_{2}$. Put

$$
W=\left\{\langle\xi, \eta\rangle \in I: \eta\left\langle f_{\alpha}(\xi)\right\} \cup\left\{f_{\xi}: \xi<\alpha\right\} .\right.
$$

If $\gamma<\alpha$ then $\left|f_{\gamma}-W\right| \leqslant \omega$, and if $\gamma>\alpha$ then $\left|f_{\gamma} \cap W\right|<\omega$, because of (8), hence $W$ is clopen. Since $\left\{f_{\xi}: \xi \in A\right\} \subseteq W$ and $|\alpha|<\omega_{1}$, and since $T$ is a $P$-space, we can easily find, using (5) (or, equivalently, (7)), disjoint open $W^{\prime}, W^{\prime \prime} \subseteq W$ such that for all $\xi<\alpha$, if $\xi \in A$ then $f_{\xi} \in W^{\prime}$ and if $\xi \notin A$ then $f_{\xi} \in W^{\prime \prime}$. Then $S=W^{\prime}$ is as required. (This way to use $<^{*}$, in a slightly different form, is due to Proctor [P].)

We now are ready to complete the proof that $V$ is as required.

FACT 4. $V$ is strongly zero-dimensional.

$\square$ Let $F$ and $G$ be disjoint closed subsets of $V$. Since $\beta \omega$ is strongly zero-dimensional there is a clopen $H \subseteq \beta \omega$ with $F \cap \beta \omega \subseteq H$ and $G \cap \beta \omega \subseteq \beta \omega-H$. Assume for a moment

(9) there is a clopen $K \subseteq V$ with $K \in \beta \omega=H$.

Then $L=(K \cup F)-G$ is a clopen set in $V$ which includes $F$ and is disjoint from $G$. $L$ is open since $K \cup F=K \cup(F \cap I)$ and $L$ is closed since $L=(K \cup F)-$ $(G \cap I)$ (recall that $I$ consists of isolated points).

It remains to prove (9). Without loss of generality $p \notin H$. Then $|H \cap C|<\omega_{1}$, hence by Fact 3 there is a clopen $S$ in $T$ with $S \cap C=H \cap C$. Then $K=H \cup S$ is clopen in $V$ since $K \cap \beta \omega=H$ is clopen in $\beta \omega$ and $K \cap T=S$ is clopen in $T$. Also, as just observed, $K \cap \beta \omega=H$. This proves (9).

FACT 5. $V$ is an $F$-space.

Let $F$ and $G$ be disjoint open $F_{o}$ 's. Let $x \in \beta \omega$ be arbitrary. Since $\beta \omega$ is an $F$-space, we have

$$
(F \cap \beta \omega)^{-} \cap(G \cap \beta \omega)^{-}=\varnothing
$$

so we may assume without loss of generality that $x \notin(F \cap \beta \omega)^{-}$. Let $U$ be a clopen neighborhood in $V$ which does not intersect $F \cap \beta \omega$ (use Fact 4). Then $F \cap U$ is an $F_{\sigma}$-subset of $V$ with $F \cap U \subseteq I(\subseteq T)$. Since $T$ is a $P$-space, it follows that $F \cap U$ is closed in $T$, consequently $F \cap U$ is closed in $V$ since $F \cap U \cap \beta \omega=\varnothing$ is closed in $\beta \omega$. Hence $x \notin \bar{F}$.

Since $x$ was arbitrary in $\beta \omega$, it follows that $\bar{F} \cap \bar{G} \cap \beta \omega=\varnothing$. As the points of $I$ are isolated this implies $\bar{F} \cap \bar{G}=\varnothing$.

FACT 6. $\beta V$ has weight $2^{\omega}$.

Since $V$ is strongly zero-dimensional and $2^{\omega_{1}}=2^{\omega}$ under $\mathrm{MA}+\neg \mathrm{CH}$ it suffices to show that $V$ has $2^{\omega_{1}}$ clopen sets. 
Since $\beta \omega$ has $2^{\omega}$ clopen sets and $|V-\beta \omega|=\omega_{1}, V$ has no more than $2^{\omega_{1}}$ clopen sets.

Recall that $I=\omega_{1} \times \omega_{1}$, and note that the way we topologize $V$ means that $\{0\} \times \omega_{1}$ is a closed discrete subset of $V$ consisting of isolated points. It follows that $V$ has at least $2^{\omega_{1}}$ clopen sets.

Remark. Perhaps the reader feels that with a more careful construction of $T$ we can ensure that $V$ has $2^{\omega}$ clopen sets without using $2^{\omega}=2^{\omega_{1}}$, but this is not the case. Let $A \subseteq C$ have cardinality $\omega_{1}$. There is a clopen set $U$ in $V$ with $U \cap C=A$ (and this property we really need). Since $T$ is a $P$-space (this property we used in an essential way in the proof of Fact 5) we can find a clopen $W_{x}$ in $T$ with $x \in W_{x}$ for $x \in A$ such that $W_{x} \subseteq U$ for $x \in A$ and $W_{x} \cap W_{y}=\varnothing$ for distinct $x, y \in A$. Choose $D \subseteq \cup_{x \in A}\left(W_{x}-\{x\}\right)$ such that $D$ intersects each $W_{x}$ in one point. Then $D$ is a closed subset of $V$ consisting of isolated points, and $|D|=\omega_{1}$.

Appendix. On normal $P$-spaces with big closed discrete sets. Fact 2 of $\$ 4$ stated that the statement

(P) there is a normal $P$-space with density $\omega_{1}$ and a closed discrete subset of cardinality $\omega_{2}$

is false under $\neg \mathrm{CH}$. (P) is similar to the statement

(Q) there is a separable normal space with an uncountable closed discrete subset.

It is a well-known result of Jones [J], (only if), and Heath [H], (if), that

(Q) if and only if $2^{\omega}=2^{\omega_{1}}$.

We use similar tools to prove our next result.

LEMMA. (P) if and only if $2^{\omega}=\omega_{1}$ and $2^{\omega_{1}}=2^{\omega_{2}}$.

Only if. Let $X$ be a $P$-space with a dense subset $D$ with $|D|=\omega_{1}$ and a closed discrete subset $C$ with $|C|=\omega_{2}$.

There are $2^{\omega_{2}}$ continuous real-valued functions on $C$ but only $2^{\omega_{1}}$ continuous real-valued functions on $D$, hence on $X$, so if $X$ is normal then $2^{\omega_{1}}=2^{\omega_{2}}$ by the Tietze-Urysohn Extension Theorem.

Now suppose $X$ is normal but $2^{\omega}>\omega_{1}$. Then there is an injection $f: C \rightarrow R$. Since $f$ is continuous and $X$ is normal, $f$ can be extended to a continuous $\bar{f}^{\leftarrow}$ : $X \rightarrow R$. For each $x \in C$ the set $f^{\leftarrow}\{f(x)\}$ is a nonempty $G_{\delta}$, hence is a nonempty open set, so $\left\{f^{\leftarrow}\{f(x)\}: x \in C\right\}$ is a disjoint open family of cardinality $\omega_{2}$. Hence $D$ is not dense. This contradiction shows that $2^{\omega}=\omega_{1}$.

If. Let $\Pi$ be the product of $2^{\omega_{1}}$ discrete two-point spaces. Let $D$ be a discrete space of cardinality $\omega_{2}$. Then the weight of $\beta D$ equals $2^{\omega_{2}}=2^{\omega_{1}}$, hence we may assume $\beta D \subseteq \Pi$.

$\Pi$ has a dense subset $A$ of cardinality $\omega_{1}$. Since $\omega_{1}^{\omega}=\omega_{1}$, there is a subset $C$ of $\Pi$ with $|C|=\omega_{1}$ such that every sequence in $A$ has a limit point in $C$.

Let $\Pi_{\delta}$ be $\Pi$, retopologized by making the $G_{\delta}$-subsets of $\Pi$ a base. It is easy to see that $C$ is dense in $\Pi_{\delta}$.

Retopologize the subspace $C \cup D$ of $\Pi_{\delta}$ by making the points of $C-D$ isolated, call the resulting space $X$. Clearly $X$ is a $P$-space in which $C$ is dense and in which $D$ is a closed discrete set. So in order to prove that $X$ is normal it suffices 
to show that disjoint subsets of $D$ have disjoint neighborhoods in $X$. Indeed, disjoint subsets of $D$ have disjoint closures in $\beta D$, hence have disjoint neighborhoods in $\Pi$, hence in $\Pi_{\delta}$, hence in $C \cup D$ as subspace of $\Pi_{\delta}$, hence in $X$.

\section{REFERENCES}

[vD ] E. K. van Douwen, $A$ basically disconnected normal space $\Phi$ with $|\beta \Phi-\Phi|=1$, Canad. J. Math. 31 (1979), 911-914.

[vD $]$, Cardinal functions on compact F-spaces and on weakly countably complete Boolean algebras (to appear).

$\left[\mathrm{vD}_{3}\right] \ldots$ Functions from the integers to the integers and topology (to appear).

[vDvM] E. K. van Douwen and J. van Mill, Parovicenko's characterization of $\beta \omega-\omega$ implies $\mathbf{C H}$, Proc. Amer. Math. Soc. 72 (1978), 539-541.

[GH] L. Gillman and M. Henriksen, Rings of continuous functions in which every finitely generated ideal is principal, Trans. Amer. Math. Soc. 82 (1956), 366-391.

[GJ] L. Gillman and M. Jerison, Rings of continuous functions, Van Nostrand, Princeton, N. J., 1960.

[H] R. W. Heath, Separability and $x_{1}$-compactness, Colloq. Math. 12 (1964), 11-14.

[J] F. B. Jones, Concerning normal and completely normal spaces, Bull. Amer. Math. Soc. 43 (1937), 671-677.

[K] S. Koppelberg, Homomorphic images of $\sigma$-complete Boolean algebras, Proc. Amer. Math. Soc. 51 (1975), 171-175.

[L] A. Louveau, Caractérisation des sous-espaces compacts de BN, Bull. Sci. Math. (2) 97 (1973), 259-263.

[MR] E. K. van Douwen, J. D. Monk and M. Rubin, Some questions about Boolean algebras, Algebra Universalis (to appear).

[P] C. W. Proctor, A separable pseudonormal nonmetrizable Moore space, Bull. Acad. Polon. Sci. Sér. Sci. Math. Astronom. Phys. 18 (1970), 179-181.

Instrtute for Medicine and Mathematics, Oho University, Athens, Oho 45701 (Curtent address of E. K. van Douwen)

Wiskundig Seminartum, Vrije Universiteit, Amsterdam, The Netherlands

Current address (J. van Mill): Department of Mathematics, Louisiana State University, Baton Rouge, Louisiana 70803 